\title{
Functional (Psychogenic) Neurological Disorders: Assessment and Acute Management in the Emergency Department
}

\author{
Jordan R. Anderson, $\mathrm{DO}^{1,2}$ Vihang Nakhate, $\mathrm{BA}^{3}$ \\ David L. Perez, MD, MMSc 3,4 \\ ${ }^{1}$ Department of Neurology, Alpert Medical School, Brown University, \\ Rhode Island Hospital, Providence, Rhode Island \\ 2 Department of Psychiatry, Alpert Medical School, Brown University, \\ Rhode Island Hospital, Providence, Rhode Island \\ ${ }^{3}$ Functional Neurology Research Group, Department of Neurology, \\ Harvard Medical School, Massachusetts General Hospital, Boston, \\ Massachusetts \\ ${ }^{4}$ Department of Psychiatry, Massachusetts General Hospital, Harvard \\ Medical School, Boston, Massachusetts
}

\author{
Christopher D. Stephen, MB ChB, MRCP3
}

Semin Neurol 2019;39:102-114.

\begin{abstract}
Address for correspondence Jordan R. Anderson, DO, Department of Neurology and Psychiatry, Butler Hospital, Providence, RI 02906 (e-mail: jordan_anderson@brown.edu).
\end{abstract}

\begin{abstract}
Keywords

- conversion disorder

- psychogenic nonepileptic seizures

- functional movement disorders

- functional paralysis

Functional neurological disorders (FND) are complex and prevalent neuropsychiatric conditions. Importantly, some patients with FND develop acute onset symptoms requiring emergency department (ED) evaluations. Historically, FND was a "rule-out" diagnosis, making assessment and management in the ED difficult. While the rapid triage of potential neurological emergencies remains the initial task, advancements have altered the approach to FND. FND is now a "rule-in" diagnosis based on validated neurological examination signs and semiological features. In this perspective article, we review signs and semiological features that can help guide the initial assessment of FND in the acute setting. Thereafter, we outline potential approaches to introduce a suspected diagnosis of FND to patients in the ED, while emphasizing the need for a comprehensive neurological evaluation. Physical and occupational therapy may be useful adjunct assessments in some individuals. Notably, clinicians in the ED setting are important members of the interdisciplinary approach to FND.
\end{abstract}

Functional neurological disorders (FND, a.k.a. conversion disorder) are increasingly recognized as highly prevalent neurobehavioral-neuropsychiatric conditions. While patients with FND are frequently seen in outpatient neurology and primary care clinics, ${ }^{1,2}$ these conditions are also common in emergency department (ED) settings. ${ }^{3}$ While large-scale epidemiologic studies of incidence and prevalence are lacking, ${ }^{4}$ there is robust evidence of high utilization of primary care, subspecialty, ED, and acute hospital services by patients with FND, resulting in considerable healthcare and societal costs. ${ }^{5}$ Notably, patients with FND show elevated repeat $\mathrm{ED}$ visit rates in comparison to other conditions. ${ }^{3} \mathrm{~A}$ variety of FND symptoms may lead to ED presentations, particularly those with paroxysmal or acute onset such as psychogenic nonepileptic seizures (PNES, a.k.a. dissociative seizures) or sensorimotor changes that mimic stroke. ${ }^{6,7}$ Other FND symptoms that may lead to ED visits include functional movement disorders (FMDs), including functional tremor, gait disturbance, and/or dystonia. Appropriate triage of FND-related cases in the ED is not yet standardized and is often complex, requiring a time-sensitive, concurrent evaluation of neurological emergencies, as well as elements of shared neurology and neuropsychiatry expertise to provide an appropriate disposition and discharge plan.

The assessment of FND has traditionally been a lengthy process utilizing tests that are not routinely performed in EDs
Issue Theme Emergency Neurology; Guest Editors, Joshua N. Goldstein, MD, $\mathrm{PhD}$, and Jeffrey M. Ellenbogen, MMSc, MD
Copyright $\odot 2019$ by Thieme Medical Publishers, Inc., 333 Seventh Avenue, New York, NY 10001, USA. Tel: +1(212) 584-4662.
DOI https://doi.org/ $10.1055 / \mathrm{s}-0038-1676844$. ISSN 0271-8235. 
(e.g., video electroencephalography [EEG]) to reach a diagnosis of exclusion. This approach discourages ED-based clinicians from considering FND in the differential diagnosis. However, FND is no longer a "diagnosis of exclusion" due to the emergence of specific physical signs and semiologic features which can be screened for and recorded during routine bedside clinical examinations. ${ }^{8-10}$ The Diagnostic and Statistical Manual of Mental Disorders was revised for its fifth edition (DSM-5) in 2013 to emphasize identifying positive features, predominantly on neurological examination, that are typical of FND. ${ }^{9}$ This revision also dropped the requirement of an acute (proximal) psychological stressor, which may not be consistently identifiable or present. Another change in the diagnostic criteria for FND was the removal of the need to explicitly exclude malingering or feigning. This is based in part on the clinical difficulties present in "ruling-out" the diagnosis of malingering, as well as consensus clinical impressions that the vast majority of patients with FND do not have clearly overlapping characteristics with the intentional falsification of symptoms for an obvious secondary gain. ${ }^{11}$ In addition, patients with FND do not generally overlap with factitious disorder (the falsification of symptoms without obvious secondary gain but rather based on an unconscious motivation, e. g., to assume the sick role).

There are many nuances to the neurobiopsychosocial formulation of FND that are beyond the scope of the ED setting (briefly discussed later and elsewhere ${ }^{10}$ ). Nevertheless, some FND patients have low adherence rates to appropriate outpatient referrals, raising the need for emergency physicians and allied clinicians to remain informed of the latest clinical understanding of FND and to advance an interdisciplinary approach to the assessment and acute management of FND. As one example, patients with FND were 10 times more likely to not show for FND-related outpatient care if newly referred from the ED in comparison to other clinical settings. ${ }^{12}$ ED clinicians should feel encouraged to entertain a suspected diagnosis of FND and empowered to communicate early diagnostic impressions to the patient where appropriate. Prior to reaching this stage, however, proper triage and workup for potential neurologic emergencies is necessary, and caution should be taken to avoid prematurely diagnosing FND.

In this perspective article, we first illustrate a typical case of an individual with FND presenting to the ED. Thereafter, we review clues in the history and bedside physical examination that can raise the index of suspicion for a FND diagnosis. We use a transdiagnostic approach in this article that cuts across the spectrum of motor FND (including functional weakness, FMD, and PNES), given that many individuals present with mixed symptoms at the time of the evaluation and those with one symptom cluster can develop new symptoms over the course of their illness. ${ }^{13,14}$ We also highlight that performance of a limited psychiatric screen may be important to detect acute precipitating mental health factors (major depression, acute suicidality, alcohol or substance misuse, etc.) that warrant concurrent triage and management. While differences in neurological training among physicians based in the ED may limit the ability to make a diagnosis of clinically established FND, the emergency room physician may be empowered to share with patients their early diagnostic impressions of a suspected FND diagnosis using transparent, nonjudgmental, and empathic language. Introducing a working diagnosis of FND to the patient, in a "rule-in" fashion as one would for other medical-neurological diagnoses, may aid patient engagement in outpatient care and allow neurologists and/ or neuropsychiatrists the opportunity to subsequently provide a definitive FND diagnosis and an individualized treatment plan. A major goal of this article is to help train clinicians based in the ED to look for, recognize, and accurately document physical examination signs and semiological features supportive of FND. This will aid a more comprehensive neurological differential diagnosis in the emergency room setting, with subsequent transition to either inpatient or outpatient care.

\section{Vignette-Mrs. A (Initial Presentation and Evaluation)}

Mrs. A. is a 34-year-old female with a history of tension-type headaches and fibromyalgia who presented to the ED with sudden onset weakness of the right arm and leg and numbness of the right face, arm, and leg that began after bumping her head at home against a cabinet. A stroke protocol was activated upon her arrival and a noncontrast head CT showed no sign of acute intracranial injury; head CT and neck angiogram were negative for large vessel occlusion. Her blood pressure was $135 / 85$, heart rate 95 , blood glucose 110. Examination revealed hyperactivation of the left lower facial musculature, moderate weakness of her right arm and leg, and downward drift (without pronation) of her right arm. Sensation to light touch was reduced on her right face with splitting at the midline, and patchy areas of numbness in her right arm and leg did not clearly follow a dermatomal distribution. She was offered tissue plasminogen activator (tPA) but declined due to concern of hemorrhage. During the discussion, she was tachypneic and diaphoretic, appeared anxious, and expressed concern that she was dying. She was given intravenous lorazepam while further workup was initiated, including a urine drug screen, basic metabolic profile, and complete blood count. When she was evaluated by a consulting neurologist 30 minutes later, her elemental neurologic exam had improved but nonetheless showed collapsing weakness in the right deltoid, a positive right Hoover sign, continued midline-splitting facial sensory loss, and an entrainable postural tremor. During the examination, the neurologist pointed out the functional examination signs to the patient-her collapsing weakness, directly right of midline sensory loss, and the modifiable rhythm of her hand tremor with volitional movements of the contralateral hand.

\section{The Clinical History in Suspected Functional Neurological Disorder}

While the clinical history is nonspecific for a diagnosis of FND, certain clues may help raise one's index of suspicion. ${ }^{15}$ With regard to FND overall (including functional weakness, 
FMD, PNES, and functional sensory symptoms), several key aspects of the history may be useful. A prodrome resembling a panic attack or dissociative experience may precede symptom onset (e.g., tachycardia, diaphoresis, dyspnea or derealization, depersonalization), even without the affective experience of panic or fear. Sudden onset with maximal severity at onset can also be seen in some individuals with FND. Patients with FND may also suffer from other functional medical comorbidities, including irritable bowel syndrome, chronic fatigue, fibromyalgia, and other chronic pain disorders. ${ }^{10}$ Neurologic comorbidities may include intellectual disability, history of mild traumatic brain injury (TBI), or epilepsy, among others. Psychiatric comorbidities may include depression, anxiety (generalized anxiety disorder or panic disorder), posttraumatic stress disorder, and personality disorders. ${ }^{16-18}$ Furthermore, a recent meta-analysis concluded that stressful life events and maltreatment are substantially more common in those with FND than in healthy controls. ${ }^{19}$ This often includes emotional neglect and sexual or physical abuse, but also other psychosocial stressors such as interpersonal difficulties. ${ }^{20}$ Notably, it is generally unnecessary to inquire about past childhood abuse in the emergency setting, unless readily disclosed by the patient in the context of a screening for active affective symptoms. While this information can be useful in the assessment and management of FND, it is generally more appropriate for inquiry in the outpatient setting with a provider who will follow up the patient longitudinally. Also, common in FND is a history of similar prior presentations with spontaneous resolution or other medically unexplained events. Thus, if ED time constraints allow, it may be appropriate to ask the patient about previous encounters with medical professionals and what impressions were communicated about the symptoms. Patients given a diagnosis of FND frequently have difficulty understanding or accepting the diagnosis, and may verbalize this as such. These elements are briefly summarized in -Table 1 . In the case of Mrs. A., history was limited based on the rapidity of stroke protocol. However, it is notable that she developed sudden unilateral sensorimotor symptoms after a triggering event of low impact (bumping her head on a cabinet), a connection that is atypical for acute stroke but has been commonly described in FND presentations. ${ }^{21}$

\section{History-Psychogenic Nonepileptic Seizures}

While PNES shares several historical features with other FND subtypes, certain features may raise the index of suspicion specifically for PNES, and may aid in distinguishing PNES from epileptic seizures (ES). Demographically, PNES patients are more likely to be female (75\%) than male and tend to first present during late adolescence to early adulthood. ${ }^{22-24}$ Though comorbid depression and anxiety are associated with PNES, these are also associated with ES and cannot be used to distinguish PNES from ES. ${ }^{25-27}$ By contrast, having a personality disorder (particularly cluster B or C) is more common in PNES vs. ES. ${ }^{26}$ Additionally, a retrospective analysis of 280 patients found that patients with PNES were more likely to have functional somatic syndromes (fibromyalgia, chronic fatigue syndrome, chronic pain syndrome, irritable bowel syndrome) and medical illnesses with chronic intermittent attacks (asthma, migraine, gastric reflux) than ES patients. ${ }^{28}$ Notably, men with PNES report significantly lower rates of physical abuse (7 vs. 32\%) and sexual abuse (3 vs. $30 \%$ ) than women. ${ }^{29}$ Finally, a history of TBI has been associated with PNES, including in military veterans. $^{30-34}$ While severe TBI may predispose to ES, mild TBI is commonly associated with PNES. ${ }^{24,31}$

\section{History-IIIness Beliefs}

Beyond common characteristics of symptom presentation, history taking may also reveal certain illness beliefs. As mentioned earlier, patients will often have seen several doctors for their symptoms. Reluctance to accept an FND diagnosis may be associated with a belief that their doctors are missing something. They may also feel strongly that a particular treatment (e.g., an antiepileptic drug) is important for their symptoms based on this belief despite evidence to the contrary. ${ }^{35}$ The challenging nature of FND diagnosis and

Table 1 Clues in the history of a possible functional neurological disorder diagnosis

\begin{tabular}{|c|c|}
\hline Historical elements & Comment \\
\hline \multirow[t]{2}{*}{$\begin{array}{l}\text { Temporal course of } \\
\text { symptom evolution }\end{array}$} & $\begin{array}{l}\text { - Sudden onset: often associated with a triggering event (such as physical injury, panic } \\
\text { attack, medical procedure, medication side effect) }\end{array}$ \\
\hline & - History of prior similar presentations with spontaneous resolutions or recurrences \\
\hline \multirow[t]{2}{*}{$\begin{array}{l}\text { Concurrent motor and } \\
\text { panic-attack features }\end{array}$} & $\begin{array}{l}\text { - Prodromal (warning) symptoms for onset including tachycardia, diaphoresis, dys- } \\
\text { pnea, and tremulousness, often without the affective experience of panic or fear }\end{array}$ \\
\hline & - Dissociative symptoms (e.g., derealization, depersonalization) \\
\hline \multirow[t]{3}{*}{$\begin{array}{l}\text { Comorbid medical and } \\
\text { psychiatric conditions }\end{array}$} & $\begin{array}{l}\text { - Medical comorbidities: irritable bowel syndrome, chronic fatigue, fibromyalgia, and } \\
\text { other chronic pain disorders }\end{array}$ \\
\hline & $\begin{array}{l}\text { - Neurological comorbidities: intellectual delay, mild traumatic brain injury, epileptic } \\
\text { seizures, migraines }\end{array}$ \\
\hline & $\begin{array}{l}\text { - Psychiatric comorbidities: depression, anxiety (generalized anxiety disorder, panic } \\
\text { disorder), posttraumatic stress disorder, and personality disorders (particularly } \\
\text { clusters B and C); common but non-specific for a functional neurological disorder }\end{array}$ \\
\hline
\end{tabular}

Source: Adapted from Stone and Carson. ${ }^{15}$ 
treatment can lead to frustration with and mistrust of the healthcare system, despite high healthcare utilization. Thus, an empathetic interviewing style is paramount to proper history taking, communication of the diagnosis, and eventual treatment planning (particularly after a time sensitive, limited triage has already occurred and the clinician can return to interview the patient in less hurried fashion). This interview style can be accomplished by taking an inventory of patient symptoms (as several symptoms are often comorbid with FND) and inquiring about functional limitations or quality of life.

\section{History-Screening for Acute Safety Concerns}

As a final note on history taking for FND in the emergency setting, psychiatric screening for high-acuity symptoms (e.g., suicidality) or other issues that could necessitate treatment considerations (e.g., drug/alcohol misuse) is generally warranted; a comprehensive psychiatric evaluation in the ED, however, may not be indicated in those who screen negative for high-acuity psychopathology. It may also be appropriate to inquire if the patient is currently in treatment with a mental health professional, as this is relevant for gauging discharge needs.

\section{Neurological Exam}

The neurological exam is the core element used to make a diagnosis of FND. ${ }^{36}$ The recognition of several neurological examination signs which can be identified at bedside has heralded the transition of FND from a "rule-out" diagnosis to a "rule-in" diagnosis. A seminal review by Daum et al sheds light on the validity of such exam elements and serves as a key reference. ${ }^{37,38}$ Exam findings can often be subtle, however, and warrant close observation and practice to avoid misdiagnosis; thus, the specificity of signs may be reduced in individuals showing "marginally" positive signs or when performed by clinicians who have more limited expertise (and confidence) in performing and interpreting neurological examination findings. Specific signs on examination for FND are described in detail later and summarized in -Table 2.

\section{Functional Motor Deficits}

The Hoover sign is based on the notion of synergistic contraction of the hip-related musculature. Normally, voluntary hip flexion performed with full effort on one side is associated with involuntary contralateral hip extension. A positive Hoover sign is identified on the supine patient in one of two ways: (1) if the patient complains of weakness of hip flexion-the examiner places a hand under the normal leg while the patient is asked to flex the "weak" leg at the hip. Absence of any hip extension (downward pressure) felt in the examiner's hand while the patient is asked to flex the contralateral side indicates functional weakness of the patient's affected side. (2) An alternative approach that highlights a cardinal element of functional weakness, internal inconsistency, can be performed by having the examiner place his/her hand under the heel of the "weak" leg, and ask the patient to first extend their "weak" leg and push down into the examiner's hand. After verifying appreciable diminished strength, the examiner can then keep his or her hand in place while also asking the patient to flex his or her contralateral normal leg (as seen in -Fig. 1). The presence of significant improved hip extension strength in the weak leg felt by the examiner indicates functional weakness. Notably, the Hoover sign can also be performed in the seated position, by placing one's hand on the top portion of one thigh and the other hand underneath their hamstring muscle to simultaneously test for hip flexion and contralateral leg extension (yielding $\quad 85.8-97.3 \%$ sensitivity and 95.7-99.9\% specificity). ${ }^{38}$

Collapsing or give-way weakness is another validated sign of functional weakness (53.9-71.5\% sensitivity, 89.4-99.1\% specificity). ${ }^{17,39,40}$ This is based on the observation that a patient can demonstrate normal strength of an extremity for a moment during the examination; however, they are unable to sustain full power and the muscle group being tested seems to "collapse" or "give-way" against the examiner's resistance during confrontation muscle testing. The test should be interpreted with caution, however, as it may also be present in patients with pain (pain-limited weakness), true fatigability, or difficulty following instructions. ${ }^{38}$ When appreciating collapsing/give-way weakness on examination, in can be helpful to ask the patients if they are in pain, as the absence of pain helps solidify the link between detection of this sign and a working diagnosis of functional limb weakness.

Motor inconsistency is another common exam finding indicative of functional weakness. Simply put, motor performance varies in two different situations testing the same muscle or muscle group. A classic example is the apparent paralysis of one leg when examined directly; however, it is noted subsequently that the patients can lift their leg to reposition themselves in bed or even stand up and cautiously ambulate independently following the examination. Similar to the Hoover sign and collapsing weakness, this is a finding with low sensitivity (2.3-41.6\%) but high specificity (85.3$99.9 \%$ ) for functional weakness. ${ }^{40}$

Hemifacial overactivity is often mistaken for facial weakness. It consists of unilateral orbicularis oris or oculus hypercontraction, accompanied by platysma contraction and jaw deviation. ${ }^{41}$ As in the vignette, Mrs. A. exhibited hyperactivation of the lower facial musculature which, given the drooping appearance, can be confused for facial weakness.

\section{Functional Sensory Deficits}

Functional sensory symptoms may be identified by neurologic examination and are often, but not exclusively, present with signs of functional weakness. Several "rule-in" signs have been identified in the literature for functional sensory deficits; however, not all are highly specific and thus they should be interpreted with caution.

Midline splitting is the loss of sensation of the entire body with a sharp demarcation at the midline, even on the trunk. 
Table 2 Specific neurological examination signs and semiological features for functional neurological disorders

\begin{tabular}{|c|c|}
\hline Functional neurological sign & Comment \\
\hline \multicolumn{2}{|l|}{ Functional weakness signs } \\
\hline Hoover sign & $\begin{array}{l}\text { Functional hip flexion: Absent contralateral hip extension when prompted to flex } \\
\text { affected leg. } \\
\text { Functional hip extension: Presence of hip extension in affected leg when prompted to } \\
\text { flex unaffected leg (best interpreted by demonstrating that initial hip extension } \\
\text { weakness on direct confrontation testing can be overcome (improved) by having the } \\
\text { patient flex their contralateral leg }\end{array}$ \\
\hline $\begin{array}{l}\text { Collapsing/Give-way } \\
\text { weakness }\end{array}$ & $\begin{array}{l}\text { Full strength evident briefly on exam, but limb collapses from normal position there- } \\
\text { after; strength suddenly gives way to sudden collapse during testing. Caution in } \\
\text { interpreting this sign in the presence of pain }\end{array}$ \\
\hline Inconsistency & $\begin{array}{l}\text { Motor performance of a muscle or muscle group varies between two tests, or between } \\
\text { objective testing and observation of motor performance }\end{array}$ \\
\hline Hemifacial overactivity & $\begin{array}{l}\text { Unilateral orbicularis oris or oculus or platysma contraction, and jaw deviation often } \\
\text { mimics an upper motor neuron pattern for facial weakness }\end{array}$ \\
\hline \multicolumn{2}{|l|}{ Functional sensory signs } \\
\hline Midline splitting & $\begin{array}{l}\text { Hemisensory loss of entire body sharply demarcated at the midline, particularly on the } \\
\text { trunk or forehead. Caution to not miss a possible thalamic lesion }\end{array}$ \\
\hline Vibratory splitting & Vibratory sensory loss sharply demarcated at midline of the frontal bone or sternum \\
\hline Nonanatomical sensory loss & $\begin{array}{l}\text { Sensory loss not conforming to known neuroanatomical distributions, e.g., anterior } \\
\text { truncal level without posterior truncal level, unilateral stocking/glove pattern }\end{array}$ \\
\hline Inconsistency & Sensory symptoms fluctuate across serial exams \\
\hline \multicolumn{2}{|c|}{ Functional movement disorder signs } \\
\hline $\begin{array}{l}\text { Tremor variability/ } \\
\text { distractibility }\end{array}$ & $\begin{array}{l}\text { Marked variability in frequency, rhythmicity, and pattern of movements; improvement, } \\
\text { pauses in tremor or resolution with distraction or when attention directed away from } \\
\text { affected limb }\end{array}$ \\
\hline Tremor entrainment & $\begin{array}{l}\text { Functional unilateral tremor adopts rhythmicity of unaffected limb during paced } \\
\text { volitional movements of unaffected limb }\end{array}$ \\
\hline Knee buckling & Knees buckle with standing or ambulation, rarely leading to falls \\
\hline Astasia-abasia & $\begin{array}{l}\text { Markedly exaggerated compensatory and uneconomical movements, often with flailing } \\
\text { arms appearing to be unstable, however, demonstrating significant degrees of pre- } \\
\text { served coordination }\end{array}$ \\
\hline Dragging monoplegic gait & $\begin{array}{l}\text { Patient with unilateral leg weakness drags leg behind them like inanimate object, often } \\
\text { with internally or externally rotated foot }\end{array}$ \\
\hline \multicolumn{2}{|c|}{ Psychogenic nonepileptic seizure semiologic features } \\
\hline Long duration & Duration over 2 min. Use with caution, as alternative is status epilepticus \\
\hline Fluctuating course & Intervening pauses, waxing/waning event tempo \\
\hline $\begin{array}{l}\text { Specific ictal movements or } \\
\text { characteristics }\end{array}$ & $\begin{array}{l}\text { Asynchronous or side-to-side movements, pelvic thrusting (can also be seen in frontal } \\
\text { lobe seizures), ictal crying }\end{array}$ \\
\hline Eye closure & Often against resistance of examiner \\
\hline Increased ictal awareness & Postictal recall of information presented ictally \\
\hline Postictal features & Absence of postseizure confusion \\
\hline Response to external stimuli & Bystanders may be able to alleviate or intensify the ictal event \\
\hline
\end{tabular}

Source: Adapted from Perez and LaFrance et al, ${ }^{6}$ McKee et al, ${ }^{10}$ Stone and Carson, ${ }^{15}$ and Daum et al. ${ }^{38}$

This is considered functional since cutaneous branches of intercostal sensory nerves overlap from the contralateral side, making sensory loss from traditional neurological conditions occurring 1 or $2 \mathrm{~cm}$ from the midline. However, one exception is in the case of thalamic lesions from stroke or tumor, which may lead to midline truncal sensory splitting. ${ }^{39,42}$
Vibratory splitting is another midline functional neurological sign, where vibration sensation is felt to differ from one side to the other and often suddenly changes at the midline when tested over a continuous bony prominence. As vibration is conducted through bone, if vibration sensation is tested on the forehead or the sternum, in general it should be perceived evenly on both sides. This test is sensitive (73.1- 


\section{Hoover Sign in Supine Position}

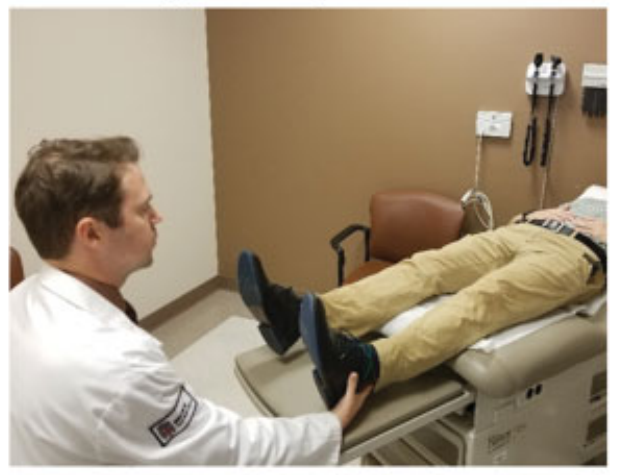

\section{Hoover Sign in Seated Position}

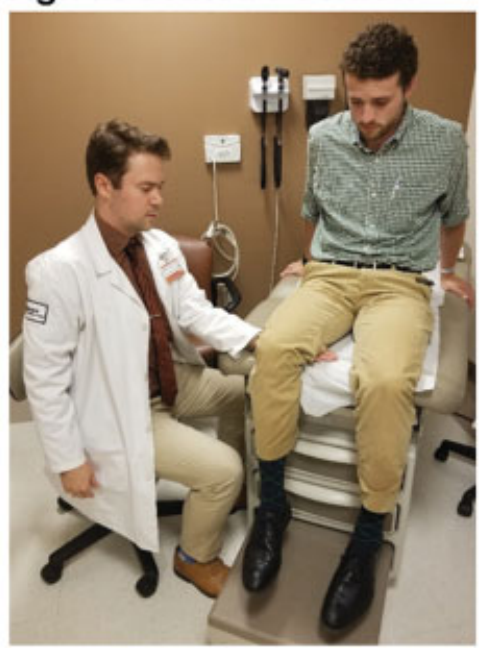

Fig. 1 Hoover sign: demonstrated in supine and sitting positions.

99.7\%) but not very specific $(7.3-23.7 \%)^{39,42,43}$ and the phenomenon has been identified in other rare neurologic conditions (ischemic or hemorrhagic stroke involving ventroposterior thalamus, ${ }^{44,45}$ corona radiata, ${ }^{46}$ paramedian dorsolateral pons, ${ }^{47}$ lenticulocapsular region, ${ }^{46,48}$ and parietal lobe ${ }^{49}$ ); therefore, it should be used with caution. ${ }^{43}$

Nonanatomical (dermatomal) sensory loss is a heterogeneous phenomenon in which patterns of patient's sensory loss do not fit a known neuroanatomical distribution. One study demonstrated $100 \%$ specificity of this finding for FND, although the finding was not well defined. ${ }^{50}$ Classically reported nondermatomal sensory loss patterns include truncal symptoms with an anterior (but not posterior) level, sharply demarcated boundaries near the groin or shoulder, or unilateral stocking/glove as seen in - Fig. 2. Inconsistency and non-reproducibility of the sensory examination findings is another commonly studied functional sensory sign. ${ }^{40,50} \mathrm{~A}$ different neurological condition such as stroke or tumor should produce relatively static sensory deficits. Logically, if sensory symptoms change throughout the exam, functional sensory loss may be considered. However, this may represent a pitfall as rapidly changing sensory symptoms may be present in conditions such as myelopathy, acute polyneuropathy, migraine, seizure, or transient ischemic attacks. $^{51}$
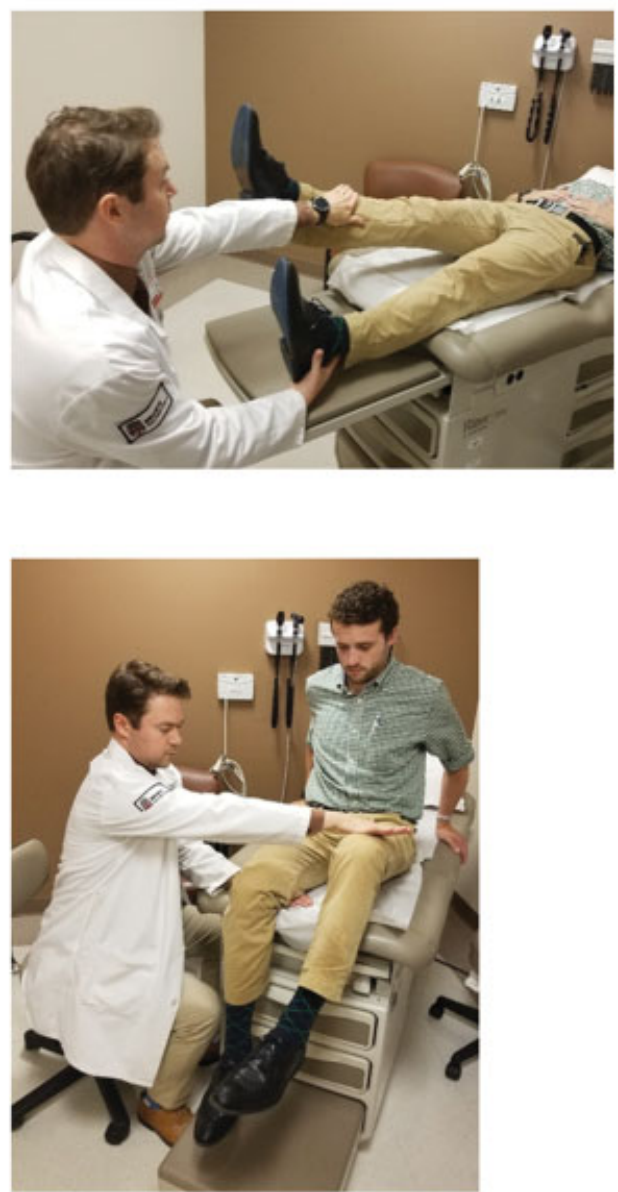

\section{Functional Movement Disorders}

FMD can be challenging to diagnose given the resemblance to other movement disorders. As with other FND subtypes, there are signs on examination to look for and attempt to elicit. These include variability and inconsistency in the frequency, rhythm and direction of tremors, abnormal movements that show features of entrainment, or resolution with distraction, among other features. ${ }^{52,53}$

Tremor variability and distractibility are specific signs for functional tremor. ${ }^{54}$ The hallmark of the movement disorder examination is visual inspection, including at rest, in specific postures, and during performance of instructed movements. Unlike patients with Parkinson's disease who show a highly reproducible resting tremor of 3 to $5 \mathrm{~Hz}$, patients with functional tremor show marked variability in the frequency and direction of abnormal movements. In addition, functional tremor often becomes more symptomatic when attention is directed toward the tremor and less so during conversation or while the patient is distracted (e.g., when asked to draw a circle with the unaffected limb). Published videos of individuals with FMD showing a highly variable and distractible tremor are available in the article by Thenganatt and Jankovic. ${ }^{55}$

Tremor entrainment is a commonly identified feature indicative of a functional tremor. Patients are asked to 


\section{Non-anatomical (Dermatomal) Sensory Loss}
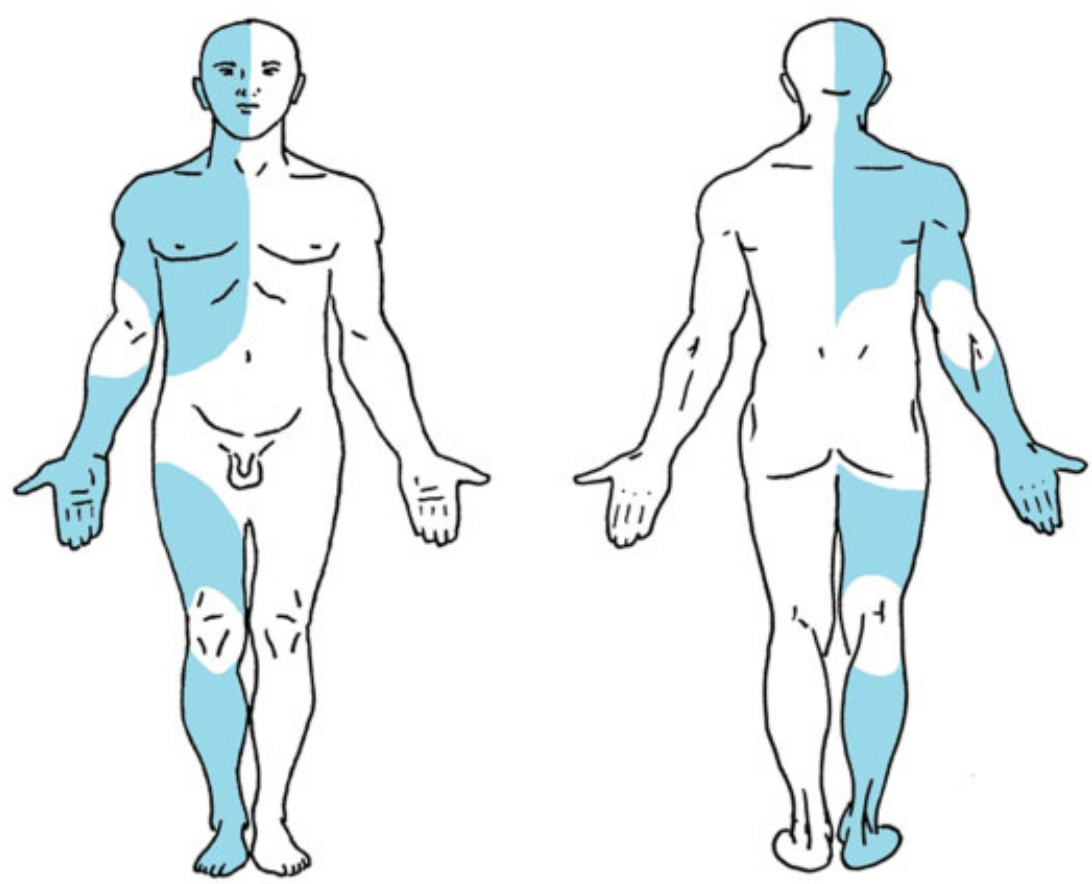

Fig. 2 Nonanatomical (dermatomal) sensory loss. Anterior and posterior view.

copy a rhythmic movement with their unaffected limb such as paced finger tapping movements or opening and closing their hand, and the affected body part adopts (or entrains to) the rhythm of volitionally performed movements in the unaffected hand. Alternatively, the affected limb ceases to exhibit abnormal movements, or the patient is unable to copy the rhythmic movement properly despite being asked to do so in the unaffected limb. Several excellent teaching videos of tremor entrainment are available by Roper and colleagues. ${ }^{56}$ Our patient (Mrs. A.) exhibited this feature during the evaluation.

FMDs may also present with abnormal gait. In the large case series of 279 patients with FMD by Baik and Lang, 36.6\% had a mixed movement disorder including a functional gait, whereas $5.7 \%$ presented as an isolated functional gait disorder. ${ }^{57}$ When presenting as a purely functional gait disorder, the most common signs include knee buckling and astasia-abasia. ${ }^{52}$ Knee buckling occurs during standing or with ambulation but rarely results in falls, while astasia-abasia is the demonstration of markedly exaggerated and uneconomical compensatory gait-related movements which, by their presence, demonstrate significant preservation of corticospinal and gait-coordination mechanisms. Caution should be used, however, with relying upon these signs as they have not been validated. ${ }^{38}$ In addition, knee buckling can be seen in patients with chorea, while astasia-abasia can rarely occur in patients with thalamic or suprathalamic lesions which cause inability to stand, also known as thalamic astasia. ${ }^{58,59}$

A dragging monoplegic gait, a subtype of functional gait disorder, can be seen in some individuals with unilateral functional leg weakness. It characteristically appears as if the leg is being dragged behind the patient like an inanimate object. The foot may be internally or externally rotated (as in -Fig. 3), and the patient may try to assist the leg during transfers to the exam table by using both hands. One study identified this sign as poorly sensitive but nearly $100 \%$ specific for functional gait. ${ }^{17}$ It can be contrasted with the hemiparetic gait that is observable in poststroke patients, which is characterized by asymmetry associated with hip extension and adduction, knee extension, and ankle plantar flexion and inversion. ${ }^{60}$

\section{Psychogenic (Dissociative) Nonepileptic Seizures}

Several studies have investigated the ictal semiology that may distinguish PNES from ES. A retrospective analysis of 34 primary studies detailed that the following clinical signs during ictal events reliably favored PNES over ES: long duration ( $>2$ minutes), fluctuating course (e.g., with intervening pauses, waxing/waning event tempo), asynchronous or side-to-side movements, pelvic thrusting, eye closure, ictal crying, postictal recall of information presented ictally (i.e., preserved ictal awareness), and absence of postictal confusion. Notably, urinary incontinence or tongue biting are nonspecific for either diagnosis. ${ }^{61,62}$ Additionally, the ability of bystanders to intensify or alleviate the event favors PNES over ES. ${ }^{63}$ That an event of longer duration is more likely to signify PNES than ES is significant, as an extended psychogenic event may be misdiagnosed as status epilepticus. ${ }^{64}$ One analysis found that an event length of 123.5 seconds was the optimal threshold to diagnose PNES, with 65\% sensitivity 


\section{Dragging Monoplegic Gait}
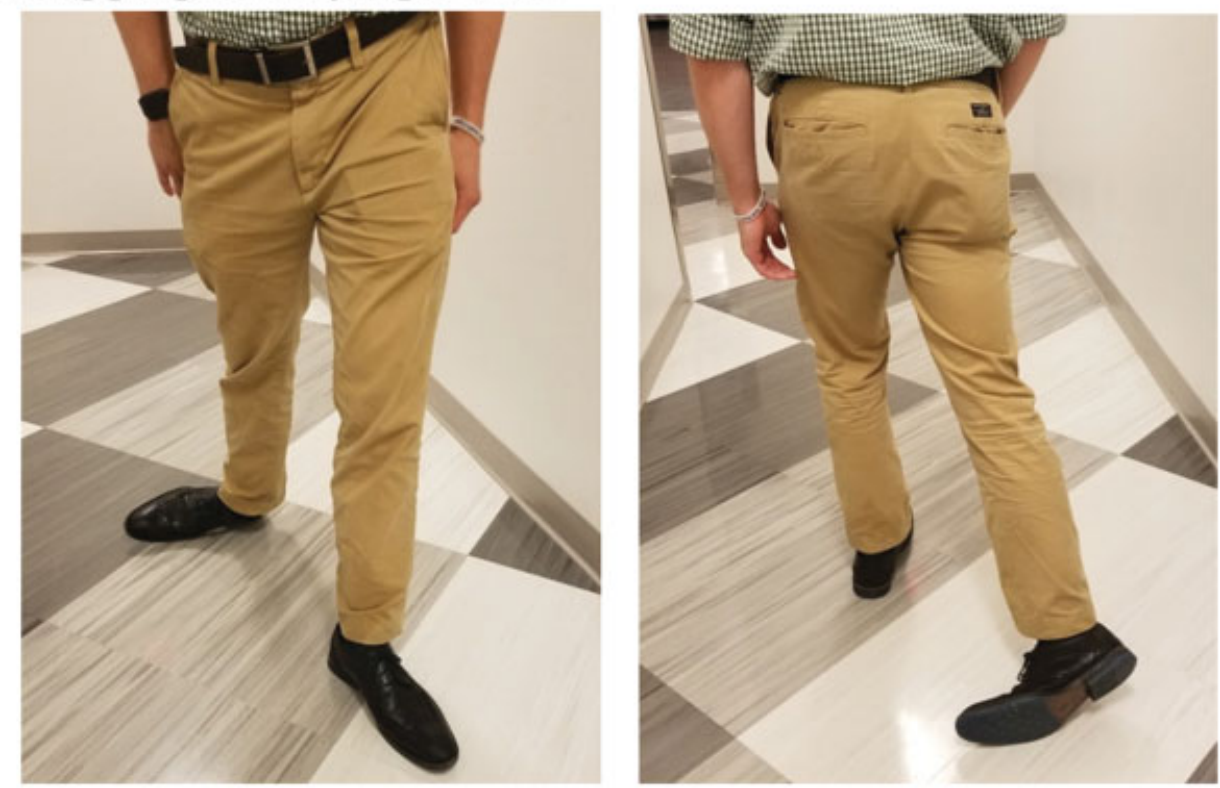

Fig. 3 Dragging monoplegic functional gait. Anterior and posterior view.

and $93 \%$ specificity. Furthermore, the authors of this study noted that if an event lasted at least 5 minutes, its odds of being PNES over ES are 24 times higher. ${ }^{62}$ This information should be used with caution; however, as an alternative to PNES in this situation is status epilepticus which is a neurological emergency. Documenting in the medical chart that an individual has a confirmed history of prolonged PNES events (nonepileptic status epilepticus) may help prevent iatrogenic injury from unnecessary urgent medical procedures such as intubation. Although some studies show that patients with PNES can have multiple stereotyped events, studies comparing PNES with ES have found that the stereotypy or variability of a patient's events is insufficient to distinguish PNES from ES. ${ }^{61,65,66}$

Most of the aforementioned semiologic features were delineated using video EEG recordings. However, when considering the diagnosis of PNES in the acute emergency setting, the best information available may be eyewitness account or first-hand observation of an event. One study prospectively interviewed eyewitnesses of seizures to document six clinical signs that distinguished PNES from ES (preserved awareness, eye flutter, modulation by bystanders for PNES; abrupt onset, eye opening/widening, and postictal confusion for ES). The authors found that eyewitness reports of these clinical signs were inaccurate and not statistically different from guessing. ${ }^{63}$ Nonetheless, a pilot study using eyewitness questionnaires found that the observation of closed eyes and side-to-side head movement by eyewitnesses was potentially highly specific (100\% specific, $66.7 \%$ sensitive) for PNES versus ES. ${ }^{67} \mathrm{~A}$ six-item semiologic bedside diagnostic tool for PNES has been proposed by a study that aimed to facilitate bedside diagnosis by first responders, taking into account the feasibility of identifying certain clinical signs in an acute setting ${ }^{68}$ (see also - Table 2).
The expertise of clinical personnel is yet another variable that affects PNES diagnostic accuracy. Empirical investigations have indicated that neurologists fare better than nonneurologists, including emergency medicine providers. ${ }^{69,70}$ Fortunately, studies also suggest that accuracy of diagnosing PNES (including by first responders and emergency providers) can be improved through targeted educational programs, by viewing more seizures on video, or otherwise by directed training in detection of relevant functional semiological features. ${ }^{68,69}$

Finally, it is worth noting that patients with epilepsy may also have psychogenic seizures, and that patients with a history of PNES can also have ES. The co-occurrence of PNES and ES is approximately $10 \%,{ }^{71}$ although estimates vary widely. ${ }^{72}$ Some patients with combined PNES/ES have ES for years before they develop a psychogenic event. Compared with patients with ES only, studies suggest that those with PNES/ES have ESs that are shorter and originate more often in the right hemisphere, in addition to higher depression and anxiety scores. $^{73}$

\section{Other Aspects of Emergency Department Evaluation}

\section{Acute Assessment Steps}

FND symptoms which prompt ED evaluations mimic other neurologic emergencies such as stroke or seizure. Such presentations should be triaged as they would normally to avoid misdiagnosis, delayed treatment initiation, and ultimately unnecessary morbidity or mortality.

\section{Stroke}

In the case of Mrs. A., a stroke protocol was appropriately activated, and she was evaluated for thrombolytic therapy in 
parallel with her evaluation for FND. It is well known that tPA administration in stroke mimics carries substantially lower risks than in true strokes. ${ }^{74}$ Thus, presentations suggestive of stroke can be treated as such when the diagnosis of FND remains clear.

\section{Seizure}

Another common situation prompting emergency treatment is seizure. Early administration of intravenous antiepileptic drugs for suspected ES is recommended by the American Epilepsy Society. ${ }^{75}$ Status epileptics has been reported in up to $30 \%$ of adults with ES, and carries substantial morbidity. While the morbidity of nonepileptic psychogenic status has scantly been studied, it is thought to be lower than that of its epileptic counterpart-and is commonly linked to iatrogenic complications. ${ }^{64}$ PNES does have several semiologic features (outlined earlier) that are commonly distinguished from ES, although some epilepsy subtypes can be mistaken for PNES. Frontal lobe epilepsy is known to present with often bizarre appearing seizures which may include bicycling movements of the legs, asynchronous movements, or pelvic thrusting. ${ }^{76}$ Therefore, withholding treatment of seizure under the suspicion of PNES should be done with significant caution (particularly if the patient has not previously received a diagnosis of PNES), despite the known morbidity of antiepileptic drugs.

\section{Communicating a "Suspected" FND Diagnosis}

When a diagnosis of FND is suspected, the physician has reached a critical point of discussion and therapeutic benefit with the patient. It is well known that FND can be perpetuated (in part) by illness beliefs, and thus lack of education about the diagnosis may contribute directly to prolonging illness. ${ }^{77}$ The physician who strongly suspects FND has the responsibility to initiate the conversation with the patient about the diagnosis and help facilitate their understanding, just as if it were any other neurologic condition. This is also to prevent the patient who receives an appropriate workup in the ED setting from leaving with the sentiment "they did all these tests that came back normal, yet they didn't tell me why I can't walk." The unique challenge of FND is that its assessment requires neurologic expertise that may not be available in the acute setting. Furthermore, neurological assessments themselves even when performed by welltrained neurologists in the ED are frequently rapid, timelimited evaluations focused on ruling-out acute neurological emergencies.

Given these challenges, it is understandable that the prospect of delivering a diagnosis of FND in the ED setting is met with some reluctance and caution. However, allowing the patient to believe that an alternative diagnosis is primarily suspected (or not receive any early-phase diagnostic impressions) may reinforce and perpetuate unhelpful illness beliefs or health fears. For individuals who have been appropriately worked up in the ED, are appropriate for home discharge and in whom positive "rule-in" signs of FND have been clearly elicited on examination, we suggest that ED physicians (in close collaboration with neurologists when possible) provide a "suspected" diagnosis of FND to patients. This allows the conversation around a possible diagnosis of FND to be initiated with the patient while also emphasizing the importance of the subsequent complete evaluation by a neurologist in the outpatient (or inpatient) setting. The documentation of clinical examination signs or semiological features supportive of an FND diagnosis is particularly important given that patients with FND can have fluctuating symptoms. For instance, some individuals who present as highly symptomatic in the ED can subsequently present as asymptomatic in the outpatient setting, requiring the outpatient neurologist to rely heavily on the documented ED exam. In addition, patients can be encouraged to video record one to two typical events when symptomatic to allow such document to be brought in to their outpatient neurology visit.

Key points to emphasize in the effective communication about the diagnosis of FND include (1) discussing the suspected diagnosis based on clinical examination; (2) providing a clear name for the condition (e.g., FND); (3) discussing with patients that this condition is common, real, and treatable. ${ }^{10,36,78}$ It may be useful to explain to the patient in simple terms the physical exam findings that led to the diagnosis, as is illustrated in our case with Mrs. A. If successful, this communication helps ensure the patients feel validated about their symptoms or functional limitations, rather than feeling unheard by providers. Additionally, confidence in the diagnosis may reduce patients' healthcare cost and enhance their engagement in outpatient referrals for further management. Several of these key points are illustrated in -Fig. 4 featuring the case of Mrs. A.

In this vignette, the emergency physician is tasked with ensuring Mrs. A. understands that the suspected diagnosis is FND, particularly because she may not have fully absorbed it when initially presented to her. A simplified analogy (software vs. hardware problem) can sometimes be useful in helping patients understand the difference between their condition and others that they are highly concerned about. The multidisciplinary approach involving the neurologist, physical therapist, and the emergency physician communicating with each other and presenting a unified message to the patient helps instill confidence in the diagnosis and willingness to move forward with treatment planning. For further education on the topic of communicating the diagnosis, helpful material is available at www.neurosymptoms. org as well as other published material. ${ }^{78}$ In addition to a physical therapy (PT) evaluation, for those with upper extremity and/or fine motor impairments, an occupational therapy evaluation if available may also be helpful to triage patients' needs. $^{79,80}$

\section{Treatment Planning}

The treatment of FND is a multidisciplinary effort that relies upon communication and collaboration among providers and the patient alike. The first step in treatment is effective communication of the diagnosis, as outlined earlier. Clinicians in the acute setting with adequate knowledge of the diagnosis should feel empowered to use this communication, especially 

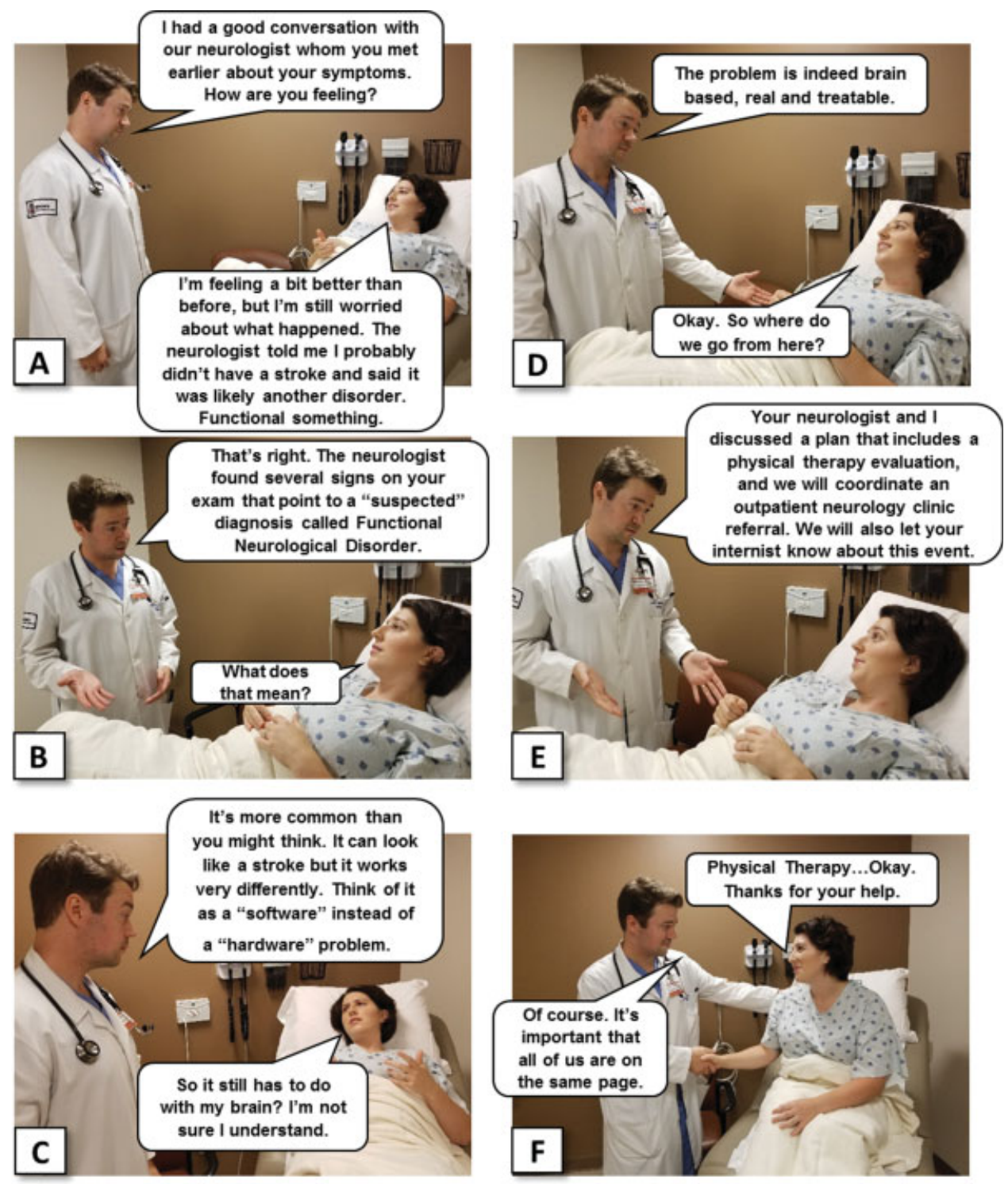

Fig. 4 Communicating a suspected functional neurological disorder diagnosis.

bolstered by a team approach. Providing printed information and further resources (such as those available from www. neurosymptoms.org) may also help reinforce the validity and patient understanding of the diagnosis. Other specialists who may commonly take part in the evaluation in the ED setting include neurologists, physical therapists, occupational therapists, psychiatrists, and social workers. Utility of specialist evaluations in the emergency setting also includes important determinations about disposition, such as inpatient admission or acute rehabilitation for those with severe motor deficits or inpatient admission for further diagnostic testing (e.g., video EEG to establish a diagnosis of documented PNES ${ }^{81}$ and further assessment of severe comorbid pain, fatigue, and cognitive difficulties that may require additional triage and workup). Notably, although a psychiatric evaluation may be helpful for those patients with significant ongoing mental health symptoms (e.g., major depression, decompensated PTSD, and suicidality), most patients do not require a comprehensive psychiatric evaluation in the ED.
In our opinion, PT assessments may be a useful adjunct to triage discharge needs for patients with functional weakness and/or functional gait. To date, literature on PT evaluations in the emergency setting for FND is lacking. However, the utility of PT in the inpatient and outpatient settings for functional motor symptoms is well established, and there are published recommendations for its use. ${ }^{10,82-84}$ Given a wide spectrum of severity, some patients may benefit from inpatient rehabilitation for severe ambulatory dysfunction, whereas others may benefit from referral to outpatient or even in-home PT evaluations. Regardless of the disposition, it is important that the physical therapist treating the patient is familiar with the consensus recommendations developed by Nielsen et al. ${ }^{84}$ If this is not the case, the patient may be subject to unnecessary supportive devices or other well-intentioned interventions that may not promote recovery. The key concepts behind the consensus recommendations include normalizing illness beliefs, education about the diagnosis, and retraining movement. Retraining movement is achieved by reducing 
selective attention to abnormal movement and demonstration that normal movement is possible. If the patient is being referred to a physical therapist who may not be familiar with these principles, it would be important for the patient to be provided with a printed version of these recommendations ${ }^{84}$ to present to their physical therapist.

Outpatient dispositions from the ED for FND symptoms are the most common. Given the complexity of FND presentations, a multidisciplinary approach should be pursued in the outpatient setting as well. Follow-up with a neurologist is important and likely a more detailed psychiatric and psychosocial screen is generally indicated to continue a thorough assessment of the patient's predisposing, precipitating and perpetuating factors. ${ }^{77}$ Ultimately, the goal of this comprehensive assessment is to develop a neurobiopsychosocial formulation to inform an individualized treatment plan. Such treatment plans may involve ongoing follow-up with neurology, psychiatry, PT, occupational therapy, psychotherapy, and/or social work. ${ }^{85}$

\section{Conclusion}

FND is a highly prevalent condition at the intersection of neurology and psychiatry, frequently presenting emergently. Increased familiarity and experience with FND by the emergency physician can further facilitate the coordinated, interdisciplinary approach that the effective assessment and management of FND demands. Identification and documentation of positive examination signs to help "rule-in" a diagnosis of FND may be a particularly helpful aspect of clinical care in the ED setting, particularly given that functional neurological symptoms can wax and wane. Future research is needed to investigate the optimal approaches to the assessment and management of patients who present to the ED with an acute FND presentation.

\section{Funding}

D.L.P. was supported by the Sidney R. Baer Jr. Foundation and the Massachusetts General Hospital Physician-Scientist Career Development Award.

\section{Disclosures/Conflicts of Interest}

D.L.P. has received honorarium for lectures from the American Academy of Neurology, Movement Disorder Society, and Harvard Medical School Continuing Education programs. All other authors do not have any conflicts of interests/disclosures.

\section{Acknowledgment}

We thank Elizabeth Nelsen and Bryan McCarthy for their assistance in -Figs. 1, 3, and $\mathbf{4}$.

\section{References}

1 Stone J, Carson A, Duncan R, et al. Who is referred to neurology clinics?-the diagnoses made in 3781 new patients Clin Neurol Neurosurg 2010;112(09):747-751

2 Stone J, Carson A, Duncan R, et al. Symptoms 'unexplained by organic disease' in 1144 new neurology out-patients: how often does the diagnosis change at follow-up? Brain 2009;132(Pt 10):2878-2888

3 Merkler AE, Parikh NS, Chaudhry S, et al. Hospital revisit rate after a diagnosis of conversion disorder. J Neurol Neurosurg Psychiatry 2016;87(04):363-366

4 Carson A, Lehn A. Epidemiology. Handb Clin Neurol 2016; 139:47-60

5 Barsky AJ, Orav EJ, Bates DW. Somatization increases medical utilization and costs independent of psychiatric and medical comorbidity. Arch Gen Psychiatry 2005;62(08):903-910

6 Perez DL, LaFrance WC Jr. Nonepileptic seizures: an updated review. CNS Spectr 2016;21(03):239-246

7 Förster A, Griebe M, Wolf ME, Szabo K, Hennerici MG, Kern R. How to identify stroke mimics in patients eligible for intravenous thrombolysis? J Neurol 2012;259(07):1347-1353

8 Stone J, LaFrance WC Jr, Brown R, Spiegel D, Levenson JL, Sharpe M. Conversion disorder: current problems and potential solutions for DSM-5. J Psychosom Res 2011;71(06):369-376

9 Stone J, LaFrance WC Jr, Levenson JL, Sharpe M. Issues for DSM-5: conversion disorder. Am J Psychiatry 2010;167(06):626-627

10 McKee K, Glass S, Adams C, et al. The inpatient assessment and management of motor functional neurological disorders: an interdisciplinary perspective. Psychosomatics 2018;59(04): 358-368

11 Galli S, Tatu L, Bogousslavsky J, Aybek S. Conversion, factitious disorder and malingering: a distinct pattern or a continuum? Front Neurol Neurosci 2018;42:72-80

12 Perez DL, Young SS, King JN, et al. Preliminary predictors of initial attendance, symptom burden, and motor subtype in a US functional neurological disorders clinic population. Cogn Behav Neurol 2016;29(04):197-205

13 Matin N, Young SS, Williams B, et al. Neuropsychiatric associations with gender, illness duration, work disability, and motor subtype in a U.S. functional neurological disorders clinic population. J Neuropsychiatry Clin Neurosci 2017;29(04):375-382

14 McKenzie PS, Oto M, Graham CD, Duncan R. Do patients whose psychogenic non-epileptic seizures resolve, 'replace' them with other medically unexplained symptoms? Medically unexplained symptoms arising after a diagnosis of psychogenic non-epileptic seizures. J Neurol Neurosurg Psychiatry 2011;82(09):967-969

15 Stone J, Carson A. Functional neurologic disorders. Continuum (Minneap Minn) 2015;21(3 Behavioral Neurology and Neuropsychiatry):818-837

16 Kranick S, Ekanayake V, Martinez V, Ameli R, Hallett M, Voon V. Psychopathology and psychogenic movement disorders. Mov Disord 2011;26(10):1844-1850

17 Stone J, Warlow C, Sharpe M. The symptom of functional weakness: a controlled study of 107 patients. Brain 2010;133(Pt 5):1537-1551

18 Roelofs K, Keijsers GP, Hoogduin KA, Näring GW, Moene FC. Childhood abuse in patients with conversion disorder. Am J Psychiatry 2002;159(11):1908-1913

19 Ludwig L, Pasman JA, Nicholson T, et al. Stressful life events and maltreatment in conversion (functional neurological) disorder: systematic review and meta-analysis of case-control studies. Lancet Psychiatry 2018;5(04):307-320

20 Reuber M, Howlett S, Khan A, Grünewald RA. Non-epileptic seizures and other functional neurological symptoms: predisposing, precipitating, and perpetuating factors. Psychosomatics 2007;48(03):230-238

21 Pareés I, Kojovic M, Pires C, et al. Physical precipitating factors in functional movement disorders. J Neurol Sci 2014;338(12):174-177

22 Chen DK, Sharma E, LaFrance WC Jr. Psychogenic non-epileptic seizures. Curr Neurol Neurosci Rep 2017;17(09):71

23 Reuber M, Brown RJ. Understanding psychogenic nonepileptic seizures - phenomenology, semiology and the integrative cognitive model. Seizure 2017;44:199-205 
24 Kerr WT, Janio EA, Braesch CT, et al. An objective score to identify psychogenic seizures based on age of onset and history. Epilepsy Behav 2018;80:75-83

25 Bowman ES, Markand ON. Psychodynamics and psychiatric diagnoses of pseudoseizure subjects. Am J Psychiatry 1996;153(01): 57-63

26 Direk N, Kulaksizoglu IB, Alpay K, Gurses C. Using personality disorders to distinguish between patients with psychogenic nonepileptic seizures and those with epileptic seizures. Epilepsy Behav 2012;23(02):138-141

27 Asmussen SB, Kirlin KA, Gale SD, Chung SS. Differences in selfreported depressive symptoms between patients with epileptic and psychogenic nonepileptic seizures. Seizure 2009;18(08): 564-566

28 Dixit R, Popescu A, Bagić A, Ghearing G, Hendrickson R. Medical comorbidities in patients with psychogenic nonepileptic spells (PNES) referred for video-EEG monitoring. Epilepsy Behav 2013; 28(02):137-140

29 Thomas AA, Preston J, Scott RC, Bujarski KA. Diagnosis of probable psychogenic nonepileptic seizures in the outpatient clinic: does gender matter? Epilepsy Behav 2013;29(02):295-297

30 Salinsky M, Spencer D, Boudreau E, Ferguson F. Psychogenic nonepileptic seizures in US veterans. Neurology 2011;77(10): 945-950

31 Salinsky M, Storzbach D, Goy E, Evrard C. Traumatic brain injury and psychogenic seizures in veterans. J Head Trauma Rehabil 2015;30(01):E65-E70

32 Westbrook LE, Devinsky O, Geocadin R. Nonepileptic seizures after head injury. Epilepsia 1998;39(09):978-982

33 Dworetzky BA, Strahonja-Packard A, Shanahan CW, Paz J, Schauble B, Bromfield EB. Characteristics of male veterans with psychogenic nonepileptic seizures. Epilepsia 2005;46(09): $1418-1422$

34 LaFrance WC Jr, Deluca M, Machan JT, Fava JL. Traumatic brain injury and psychogenic nonepileptic seizures yield worse outcomes. Epilepsia 2013;54(04):718-725

35 LaFrance WC Jr, Reuber M, Goldstein LH. Management of psychogenic nonepileptic seizures. Epilepsia 2013;54(Suppl 1):53-67

36 Espay AJ, Aybek S, Carson A, et al. Current concepts in diagnosis and treatment of functional neurological disorders. JAMA Neurol 2018;75(09):1132-1141

37 Daum C, Gheorghita F, Spatola M, et al. Interobserver agreement and validity of bedside 'positive signs' for functional weakness, sensory and gait disorders in conversion disorder: a pilot study. J Neurol Neurosurg Psychiatry 2015;86(04):425-430

38 Daum C, Hubschmid M, Aybek S. The value of 'positive' clinical signs for weakness, sensory and gait disorders in conversion disorder: a systematic and narrative review. J Neurol Neurosurg Psychiatry 2014;85(02):180-190

39 Gould R, Miller BL, Goldberg MA, Benson DF. The validity of hysterical signs and symptoms. J Nerv Ment Dis 1986;174(10): 593-597

40 Chabrol H, Peresson G, Clanet M. Lack of specificity of the traditional criteria for conversion disorders. Eur Psychiatry 1995;10(06):317-319

41 Fasano A, Valadas A, Bhatia KP, et al. Psychogenic facial movement disorders: clinical features and associated conditions. Mov Disord 2012;27(12):1544-1551

42 Rolak LA. Psychogenic sensory loss. J Nerv Ment Dis 1988;176 (11):686-687

43 Toth C. Hemisensory syndrome is associated with a low diagnostic yield and a nearly uniform benign prognosis. J Neurol Neurosurg Psychiatry 2003;74(08):1113-1116

44 Bogousslavsky J, Regli F, Uske A. Thalamic infarcts: clinical syndromes, etiology, and prognosis. Neurology 1988;38(06): 837-848

45 Paciaroni M, Bogousslavsky J. Pure sensory syndromes in thalamic stroke. Eur Neurol 1998;39(04):211-217
46 Kim JS. Pure sensory stroke. Clinical-radiological correlates of 21 cases. Stroke 1992;23(07):983-987

$47 \mathrm{Kim}$ JS, Bae YH. Pure or predominant sensory stroke due to brain stem lesion. Stroke 1997;28(09):1761-1764

$48 \mathrm{Kim}$ JS. Lenticulocapsular hemorrhages presenting as pure sensory stroke. Eur Neurol 1999;42(03):128-131

49 Bassetti C, Bogousslavsky J, Regli F. Sensory syndromes in parietal stroke. Neurology 1993;43(10):1942-1949

50 Baker JH, Silver JR. Hysterical paraplegia. J Neurol Neurosurg Psychiatry 1987;50(04):375-382

51 Nadarajan V, Perry RJ, Johnson J, Werring DJ. Transient ischaemic attacks: mimics and chameleons. Pract Neurol 2014;14(01):23-31

52 Barbey A, Aybek S. Functional movement disorders. Curr Opin Neurol 2017;30(04):427-434

53 Hallett M. Functional (psychogenic) movement disorders - clinical presentations. Parkinsonism Relat Disord 2016;22(Suppl 1): S149-S152

54 Kenney C, Diamond A, Mejia N, Davidson A, Hunter C, Jankovic J. Distinguishing psychogenic and essential tremor. J Neurol Sci 2007;263(1-2(:94-99

55 Thenganatt MA, Jankovic J. Psychogenic tremor: a video guide to its distinguishing features. Tremor Other Hyperkinet Mov (N Y) 2014;4:253

56 Roper LS, Saifee TA, Parees I, Rickards H, Edwards MJ. How to use the entrainment test in the diagnosis of functional tremor. Pract Neurol 2013;13(06):396-398

57 Baik JS, Lang AE. Gait abnormalities in psychogenic movement disorders. Mov Disord 2007;22(03):395-399

58 Elwischger K, Rommer P, Prayer D, Mueller C, Auff E, Wiest G. Thalamic astasia from isolated centromedian thalamic infarction. Neurology 2012;78(02):146-147

59 Takahashi K, Osaka A, Tsuda H, Ogasawara H. Isolated astasia caused by a localized infarction in the suprathalamic white matter. J Gen Fam Med 2017;18(05):275-278

60 Sheffler LR, Chae J. Hemiparetic Gait. Phys Med Rehabil Clin N Am 2015;26(04):611-623

61 Avbersek A, Sisodiya S. Does the primary literature provide support for clinical signs used to distinguish psychogenic nonepileptic seizures from epileptic seizures? J Neurol Neurosurg Psychiatry 2010;81(07):719-725

62 Seneviratne U, Minato E, Paul E. How reliable is ictal duration to differentiate psychogenic nonepileptic seizures from epileptic seizures? Epilepsy Behav 2017;66:127-131

63 Syed TU, LaFrance WC Jr, Kahriman ES, et al. Can semiology predict psychogenic nonepileptic seizures? A prospective study. Ann Neurol 2011;69(06):997-1004

64 Dworetzky BA, Weisholtz DS, Perez DL, Baslet G. A clinically oriented perspective on psychogenic nonepileptic seizure-related emergencies. Clin EEG Neurosci 2015;46(01):26-33

65 Herskovitz M. Stereotypy of psychogenic nonepileptic seizures. Epilepsy Behav 2017;70(Pt A):140-144

66 Asadi-Pooya AA, Tinker J, Fletman EW. How variable are psychogenic nonepileptic seizures? A retrospective semiological study. J Neurol Sci 2017;377:85-87

67 Erba G, Bianchi E, Giussani G, Langfitt J, Juersivich A, Beghi E. Patients' and caregivers' contributions for differentiating epileptic from psychogenic nonepileptic seizures. Value and limitations of self-reporting questionnaires: a pilot study. Seizure 2017; 53:66-71

68 De Paola L, Terra VC, Silvado CE, et al. Improving first responders' psychogenic nonepileptic seizures diagnosis accuracy: development and validation of a 6 -item bedside diagnostic tool. Epilepsy Behav 2016;54:40-46

69 Jin B, Wu H, Xu J, et al. Analyzing reliability of seizure diagnosis based on semiology. Epilepsy Behav 2014;41:197-202

70 Wasserman D, Herskovitz M. Epileptic vs psychogenic nonepileptic seizures: a video-based survey. Epilepsy Behav 2017; 73:42-45 
71 Duncan R, Oto M. Predictors of antecedent factors in psychogenic nonepileptic attacks: multivariate analysis. Neurology 2008;71 (13):1000-1005

72 Reuber M, Qurishi A, Bauer J, et al. Are there physical risk factors for psychogenic non-epileptic seizures in patients with epilepsy? Seizure 2003;12(08):561-567

73 Wissel BD, Dwivedi AK, Gaston TE, et al. Which patients with epilepsy are at risk for psychogenic nonepileptic seizures (PNES)? A multicenter case-control study. Epilepsy Behav 2016;61:180-184

74 Chernyshev OY, Martin-Schild S, Albright KC, et al. Safety of tPA in stroke mimics and neuroimaging-negative cerebral ischemia. Neurology 2010;74(17):1340-1345

75 Glauser T, Shinnar S, Gloss D, et al. Evidence-based guideline: treatment of convulsive status epilepticus in children and adults: report of the Guideline Committee of the American Epilepsy Society. Epilepsy Curr 2016;16(01):48-61

76 Beleza P, Pinho J. Frontal lobe epilepsy. J Clin Neurosci 2011;18 (05):593-600

77 Baslet G, Seshadri A, Bermeo-Ovalle A, Willment K, Myers L. Psychogenic non-epileptic seizures: an updated primer. Psychosomatics 2016;57(01):1-17

78 Carson A, Lehn A, Ludwig L, Stone J. Explaining functional disorders in the neurology clinic: a photo story. Pract Neurol 2016;16(01):56-61
79 Gardiner P, MacGregor L, Carson A, Stone J. Occupational therapy for functional neurological disorders: a scoping review and agenda for research. CNS Spectr 2018;23(03):205-212

80 Ranford J, Perez DL, MacLean J. Additional occupational therapy considerations for functional neurological disorders: a potential role for sensory processing. CNS Spectr 2018;23(03):194-195

81 LaFrance WC Jr, Baker GA, Duncan R, Goldstein LH, Reuber M. Minimum requirements for the diagnosis of psychogenic nonepileptic seizures: a staged approach: a report from the International League Against Epilepsy Nonepileptic Seizures Task Force. Epilepsia 2013;54(11):2005-2018

82 Jacob AE, Kaelin DL, Roach AR, Ziegler CH, LaFaver K. Motor retraining (MoRe) for functional movement disorders: outcomes from a 1-week multidisciplinary rehabilitation program. PM R 2018; doi: 10.1016/j.pmrj.2018.05.011

83 Nielsen G, Stone J, Edwards MJ. Physiotherapy for functional (psychogenic) motor symptoms: a systematic review. J Psychosom Res 2013;75(02):93-102

84 Nielsen G, Stone J, Matthews A, et al. Physiotherapy for functional motor disorders: a consensus recommendation. J Neurol Neurosurg Psychiatry 2015;86(10):1113-1119

85 Adams C, Anderson J, Madva EN, LaFrance WC Jr, Perez DL. You've made the diagnosis of functional neurological disorder: now what? Pract Neurol 2018;18(04):323-330 